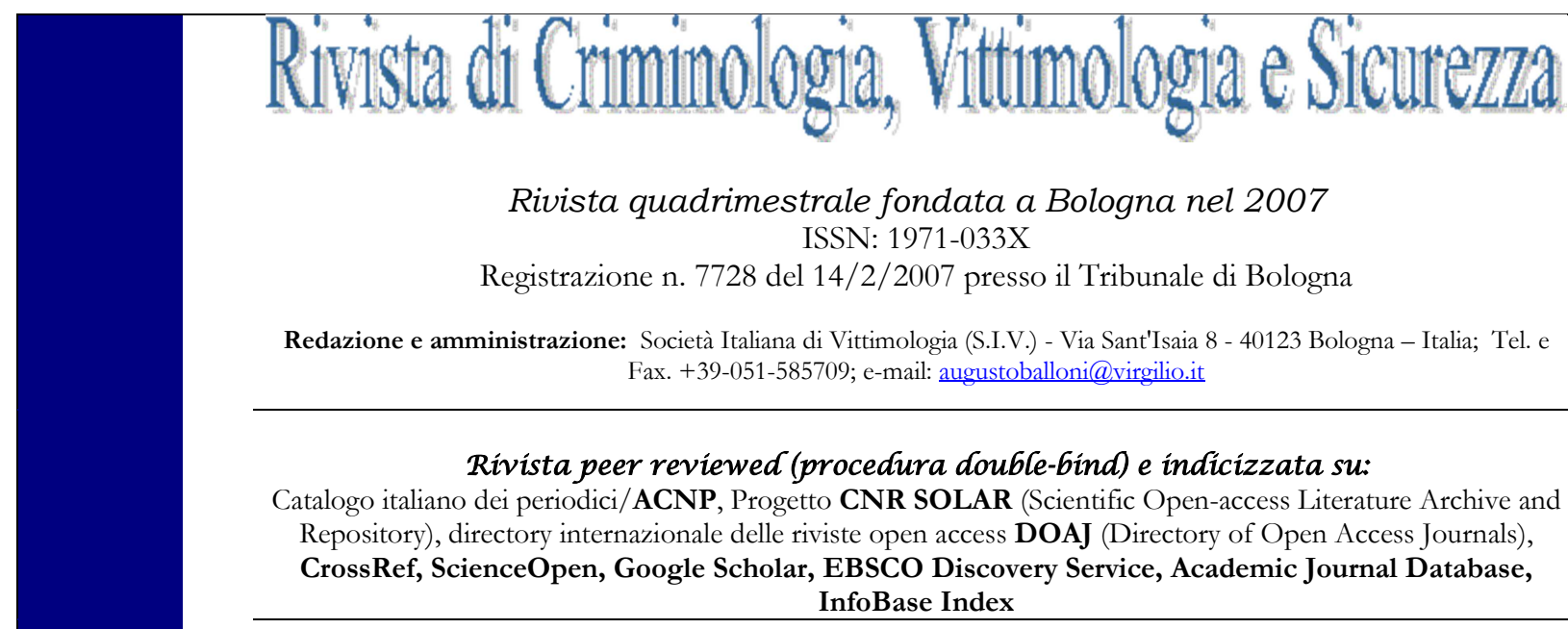

Tutti gli articoli pub6licati su questa Rivista sono distribuiti con licenza Creative Commons Attribution-NonCommercial-NoDerivatives 4.0 International Public License (cc) EY-NC-ND

Editore e Direttore:

Augusto BALLONI, presidente S.I.V., già professore ordinario di criminologia, Università di Bologna, Italia (direzione@,vittimologia.it)

\title{
COMITATO EDITORIALE
}

Coordinatore:

Raffaella SETTE, dottore di ricerca in criminologia, professore associato, Università di Bologna, Italia (redazione@,vittimologia.it)

Elena BIANCHINI (Università di Bologna), Roberta BIOLCATI (Università di Bologna), Fabio BRAVO (Università di Bologna), Lorenzo Maria CORVUCCI (Foro di Bologna), Emilia FERONE (Università "G. D’Annunzio", Chieti-Pescara), Francesco FERZETTI (Università “G. D’Annunzio”, Chieti-Pescara), Maria Pia GIUFFRIDA (Associazione Spondé), Giorgia MACILOTTI (Università Tolosa 1 Capitole, Francia), Andrea PITASI (Università "G. D'Annunzio, Chieti-Pescara), Sandra SICURELLA (Università di Bologna)

\section{COMITATO SCIENTIFICO}

Coordinatore:

Roberta BISI, vice Presidente S.I.V., professore ordinario di sociologia della devianza, Università di Bologna, Italia (comitatoscientifico@,vittimologia.it)

Andrea BIXIO (Università Roma "La Sapienza"), Encarna BODELON (Università Autonoma di Barcellona, Spagna), Stefano CANESTRARI (Università di Bologna), Laura CAVANA (Università di Bologna), Gyorgy CSEPELI (Institute of Advanced Studies Koszeg, Ungheria), Janina CZAPSKA (Università Jagiellonian, Cracovia, Polonia), Lucio D'ALESSANDRO (Università degli Studi Suor Orsola Benincasa, Napoli), François DIEU (Università Tolosa 1 Capitole, Francia), Maria Rosa DOMINICI (S.I.V.), John DUSSICH (California State University, Fresno), Jacques FARSEDAKIS (Università Europea, Cipro), André FOLLONI (Pontifical Catholic University of Paraná, Brasile), Ruth FREEMAN (University of Dundee, UK), Paul FRIDAY (University of North Carolina, Charlotte), Shubha GHOSH (Syracuse University College of Law, USA), Xavier LATOUR (Université Côte d'Azur), Jean-Marie LEMAIRE (Institut Liégeois de Thérapie Familiale, Belgio), André LEMAÎTRE (Università di Liegi, Belgio), Silvio LUGNANO (Università degli Studi Suor Orsola Benincasa, Napoli), Mario MAESTRI (Società Psicoanalitica Italiana, Bologna), Luis Rodriguez MANZANERA (Università Nazionale Autonoma del Messico), Gemma MAROTTA (Sapienza Università di Roma), Vincenzo MASTRONARDI (Unitelma-Sapienza, Roma), Maria Rosa MONDINI (Centro Italiano di Mediazione e Formazione alla Mediazione, Bologna), Stephan PARMENTIER (Unviersità Cattolica, Lovanio, Belgio), Tony PETERS† (Università Cattolica, Lovanio, Belgio), Monica RAITERI (Università di Macerata), Francesco SIDOTI (Università de l'Aquila), Philip STENNING (Università di Griffith, Australia), Liborio STUPPIA (Università "G. D'Annunzio, Chieti-Pescara), Emilio VIANO (American University, Washington, D.C.), Sachio YAMAGUCHI (Università Nihon Fukushi, Giappone), Simona ZAAMI (Università Roma "La Sapienza"), Christina ZARAFONITOU (Università Panteion, Atene), Vito ZINCANI (Procura della Repubblica, Modena), Vladimir ZOLOTYKH (Udmurt State University, Russia) 


\title{
Il Minnesota Multiphasic Personality Inventory-2 nel contesto forense: studio su coppie di genitori in fase di separazione ed affidamento minori
}

\author{
L'Inventaire Multiphasique de Personnalité du Minnesota-2 dans le contexte \\ juridique : \\ une étude sur les parents confrontés à une séparation et à la garde des enfants
}

\section{The Minnesota Multiphasic Personality Inventory-2 Test in the legal context: a study on parents going through separation and the custody of children}

\author{
Michele Lasala, Roberto Cicioni, Tommaso Caravelli, Flora Masini
}

\begin{abstract}
Riassunto
Obiettivo di questo lavoro è analizzare i profili medi di genitori valutati in sede di consulenza tecnica per l'affidamento di figli minori attraverso il Minnesota Multiphasic Personality Inventory 2 (MMPI-2), il questionario di personalità maggiormente utilizzato in ambito giuridico.

Si tratta di uno studio iniziale ed esplorativo, condotto su un campione di 200 periziandi divisi equamente tra uomini e donne, che si propone in primis di rispondere all'esigenza dello psicodiagnosta forense di avere dati statistici specifici a cui far riferimento quando utilizza tale strumento.

Il lavoro ha anche l'obiettivo di osservare la presenza di eventuali differenze significative tra i dati emersi dal campione peritale ed i valori normativi generali della popolazione italiana.
\end{abstract}

\section{Résumé}

Le but de cette étude est celui d'analyser les profils moyens des parents évalués par le test MMPI-2 (Inventaire Multiphasique de Personnalité du Minnesota-2) afin d'obtenir la garde de leurs enfants. Le MMPI-2 est le questionnaire de personnalité le plus utilisé dans le contexte juridique.

Dans l'article, les auteurs présentent une étude initiale et exploratoire menée sur un échantillon de 200 personnes, réparties de manière égale entre hommes et femmes.

En outre, cette étude a pour objet d'observer les différences statistiquement significatives entre l'échantillon et la population de référence. En effet, elle montre certaines différences entre les valeurs moyennes dans ces deux groupes, notamment entre les deux sexes.

\section{Abstract}

The aim of this study is to analyse the average profiles of parents evaluated through the MMPI-2 test (Minnesota Multiphasic Personality Inventory 2) in order to obtain custody of their children. The MMPI-2 is a psychological test that assesses personality traits which is the most used test in legal context.

This is an initial and exploratory study done on a sample of 200 people, equally divided between men and women, assessed with a view to obtaining custody of a child.

In addition, the purpose of this study is to observe statistical significant differences between the sample and the reference population. Our study shows some differences between the mean values in these two different groups, particularly some specific differences between the sexes.

Key words: MMPI-2; psicodiagnostica forense; affidamento figli minori.

\footnotetext{
- Michele Lasala è psicologo, psicoterapeuta, docente Istituto Rorschach Forense, terapeuta EMDR, socio Società Italiana di Terapia Comportamentale e Cognitiva e Centro di Psicoterapia Cognitivo Comportamentale; Roberto Cicioni è psicologo e direttore Istituto Rorschach Forense; Tommaso Caravelli è psicologo e presidente Istituto Rorschach Forense; Flora Masini è psicologa, psicoterapeuta, terapeuta EMDR, socia Società Italiana di Terapia Comportamentale e Cognitiva e Centro di Psicoterapia Cognitivo Comportamentale.
} 


\section{Introduzione.}

All'interno della Consulenza Tecnica d'Ufficio (C.T.U.) il ruolo dello psicodiagnosta forense è quello di delineare, nel modo più dettagliato e preciso possibile, le caratteristiche di personalità del periziando maggiormente rilevanti per gli scopi della valutazione, ossia di rendere disponibile ai Consulenti ed al Magistrato un quadro coerente ed articolato delle eventuali problematiche e difficoltà e delle risorse a loro potenziale bilanciamento.

Esistono varie correnti di pensiero circa l'utilità dei test per ottenere informazioni sulla personalità del periziando (1): alcuni professionisti, soprattutto chi svolge attività psicoterapeutica, li considerano scarsamente utili o superflui e tendono a prediligere i colloqui; al contrario, altri professionisti li considerano dei validi strumenti di ausilio ai colloqui (2).

Nonostante non sempre nel percorso di una CTU sia necessario sottoporre il periziando (o i periziandi) alla somministrazione di test e questionari (ㄱ), chi scrive ritiene il loro utilizzo molto importante in quanto essi permettono di ottenere dei dati maggiormente oggettivi rispetto a quelli che emergono dai colloqui e che devono necessariamente essere integrati con questi allo scopo di avere una visione più ampia ed analitica della personalità dei periziandi (4).

In ambito peritale $i$ test maggiormente utilizzati sono i test di personalità. Essi sono così chiamati in quanto permettono di delineare il profilo della personalità del soggetto a cui vengono somministrati, consentendo di avere in poco tempo delle informazioni dettagliate sugli aspetti cognitivi, affettivi e relazionali del periziando.

I test e questionari di personalità possono essere classificati in vari modi differenti. Essi vengono divisi in monofasici e multifasici: i primi misurano un solo aspetto della personalità dell'individuo; i secondi indagano più dimensioni contemporaneamente.

Ancora, essi possono essere distinti in test proiettivi e test non proiettivi: $i$ primi sono così chiamati in quanto si parte dall'assunto che durante la loro somministrazione il soggetto, inconsapevolmente, proietta parti della propria personalità su degli stimoli solitamente poco strutturati e definiti (es. durante la visione di macchie di inchiostro o durante la produzione di una figura umana o di un albero, ecc. - dipende dal test nello specifico a cui il soggetto è sottoposto); i secondi, al contrario, solitamente consistono in una serie di domande a cui il soggetto deve rispondere $\mathrm{O}$ in modo dicotomico (Si/No) o su una scala Likert da 1 a 5 o da 1 a 7 punti, dove solitamente il punteggio più basso indica l'assenza di un determinato aspetto indagato dalla domanda, mentre il punteggio più alto indica la presenza massiccia di tale aspetto.

Chi scrive ritiene maggiormente oggettivi i dati che emergono dall'utilizzo di strumenti standardizzati rispetto a quelli che emergono dai colloqui clinici in quanto nei test e nei questionari i risultati vengono confrontati con quelli presenti nella letteratura di riferimento. Al contrario, le informazioni raccolte durante $\mathrm{i}$ colloqui hanno una valenza soprattutto qualitativa ( $(\underline{5})$ e potrebbero risentire delle caratteristiche cognitive ed affettive di chi ascolta e raccoglie le informazioni.

A tal proposito, però, un discorso più approfondito va fatto per l'utilizzo dei test proiettivi, poiché anche durante il loro impiego le caratteristiche di personalità dello psicodiagnosta potrebbero avere un'importante influenza sulla tipologia dei dati raccolti e sulla loro interpretazione. In base a quanto 
appena detto è fondamentale che lo psicodiagnosta abbia una rigorosa formazione specifica circa lo strumento che utilizza ed una notevole esperienza nella somministrazione ed interpretazione. Se confermati entrambi questi elementi, si riducono drasticamente imprecisioni ed errori che, solitamente, quando presenti, sono ascritti ai test utilizzati, mentre rappresentano più spesso l'esito dellimprovvisazione o scarsa preparazione dello psicodiagnosta.

In ambito peritale $i$ test di personalità maggiormente utilizzati sono il Minnesota Multiphasic Personality Inventory 2 (MMPI-2) ed il test di Rorschach (ㅁ).

In casi di separazione ed affidamento minori, è molto utilizzato anche il Millon Clinical Multiaxal Iventory (MCMI) (ㄱ) giunto attualmente alla III edizione.

Solitamente i periziandi risultano mediamente più prudenti, diffidenti e talvolta addirittura ostili nei confronti dei test, anche perché è frequente e normale che test e questionari attivino vissuti di ansia. In ogni caso si tratta sempre di atteggiamenti che non seguono il principio tutto/nulla, visto che ogni periziando si posiziona in maniera differente lungo un continuum che può andare da una forma lieve di chiusura ad altre molto più marcate. In tutti i casi si tratta di atteggiamenti che facilmente sfociano in una riduzione del numero di informazioni. La consapevolezza di questi aspetti è un elemento che lo psicodiagnosta deve tenere in grande considerazione nel colloquio che precede la somministrazione, durante il quale deve sapientemente accogliere e contenere le normali ansie del periziando, consentendogli di superare le lecite resistenze, al fine di motivarlo e predisporlo a fornire informazioni su se stesso. Un approccio col periziando poco disponibile o rigido, comunque non ricettivo verso questi impliciti vissuti dei periziandi, può incidere seriamente sui risultati dei test (8).

Nel caso specifico del MMPI-2, la forma di chiusura più marcata è definita faking good (9), letteralmente "apparire buoni". Si tratta di un comportamento distorsivo (10), definito anche "profilo dissimulatorio" (11) in cui le scale L e K (due tra le scale di Validità del test) hanno entrambe un punteggio $\mathrm{T} \geq 70 \mathrm{ed} F$ (un'altra scala di Validità) un punteggio $\mathrm{T}<50$. La presenza di un profilo dissimulatorio molto spesso causa punteggi moderatamente bassi nella maggior parte delle scale di base rispetto ai valori normativi (12).

Oltre al profilo dissimulatorio, in letteratura si parla anche di "profilo difensivo" (13): anche in questo caso l'individuo mostra una tendenza a presentarsi in maniera irrealisticamente favorevole, tuttavia tale atteggiamento è meno marcato rispetto al precedente ed è caratterizzato dalle scale L-K entrambe più elevate della scala $F$, ma con un punteggio compreso tra $60 \leq \mathrm{T} \leq 69$ (dunque con elevazioni inferiori rispetto al profilo dissimulatorio). Anche la presenza di un profilo difensivo può chiaramente causare punteggi moderatamente bassi nelle scale di base, pur se non dovrebbero abbassarsi come nel profilo dissimulatorio.

\section{Metodologia.}

\subsection{Campione.}

Il campione di questo studio è di 200 soggetti, valutati in ambito peritale durante Consulenze Tecniche per l'affidamento dei figli in casi di separazioni, equamente distribuiti per sesso e con età compresa tra 29-56 anni (età media 43 anni; 45 anni per il gruppo degli uomini, 42 anni per il gruppo delle donne). La maggior parte dei soggetti ha un livello di istruzione pari alla licenza media 
superiore e quasi tutti provengono dal Nord e Centro Italia. Può essere un elemento utile da considerare il fatto che gli MMPI-2 sono stati tutti compilati successivamente ad un colloquio iniziale di accoglienza ed alla somministrazione del Test di Rorschach.

\subsection{Strumento.}

Lo strumento utilizzato è il MMPI-2, questionario self report ad ampio spettro, composto da 567 item a cui il soggetto può rispondere in modo dicotomico "Vero/Falso". Tutte le risposte fornite al questionario confluiscono poi in una serie di scale, ognuna delle quali avrà una propria elevazione: per ogni scala le risposte vengono tradotte in punteggi, prima grezzi e poi ponderati T; unendo tali elevazioni si ottengono vari grafici.

Ideato dallo psicologo S.R. Hathaway e dal neuropsichiatra J.C. McKinley e pubblicato per la prima volta poco dopo il 1940, nel 1989, dopo un accurato lavoro di revisione, viene pubblicato il MMPI-2 ad opera di J. N. Butcher et al.

In Italia l'adattamento fu pubblicato ad opera di Pancheri e Sirigatti nel 1995 in seguito ad una taratura effettuata su 1375 soggetti italiani, 403 maschi e 972 femmine (14).

Ad oggi è l'inventario self-report standardizzato più conosciuto ed utilizzato: in letteratura esistono oltre 14.000 pubblicazioni tra monografie e articoli specialistici sul MMPI-2.

Si tratta di uno degli strumenti più utili e validi da un punto di vista empirico, in grado di fornire una valutazione quantitativa e qualitativa delle caratteristiche della personalità dell'individuo (15). Esso, inoltre, permette di avere un quadro chiaro sia delle difficoltà del soggetto al momento della somministrazione, sia delle sue risorse da un punto di vista cognitivo ed affettivo-relazionale.
Il cut-off per tutte le scale è 65 (considerando che per ogni scala la media è 50 , il punteggio 65 corrisponde al $92^{\circ}$ percentile del campione su cui è basata la taratura; solo l'8\% del campione aveva punteggi $\mathrm{T} \geq$ 65 nelle scale del questionario): elevazioni $\mathrm{T} \geq 65$ sono significative ed indicative della presenza di difficoltà patologiche relativamente all'area indagata dalla specifica scala.

Di seguito viene presentata una sintesi delle diverse scale:

- 7 Scale di Validità: ? ("Non so"), L (Lie), F (Infrequency), K (Correction), Vrin (Variable Response Inconsistency), Trin (True Response Inconsistency), $\mathrm{Fb}$ (Back Infrequency). Esse sono così chiamate in quanto danno informazioni circa la validità del profilo emerso. Si tratta delle prime scale da osservare poiché la loro elevazione e configurazione permettono di individuare sin da subito l'eventuale presenza nel soggetto di atteggiamenti durante la compilazione del questionario che potrebbero aver interferito con le informazioni riferite ed emerse sia in termini di quantità, che in termini di intensità, frequenza e pervasività. Solo dopo aver compreso se il profilo emerso dalle varie scale è da considerarsi valido o meno (non è sempre facile giungere ad una conclusione certa), lo psicodiagnosta è in grado di decidere se proseguire, o meno, con l'interpretazione delle varie scale ed in che modo trattare le varie informazioni emerse;

- 10 scale di Base: 1.Hs (Ipocondria), 2.D (Depressione), 3.Hy (Isteria), 4.Pd (Deviazione Psicopatica), 5.Mf (Mascolinità/Femminilità), 6.Pa (Paranoia), 7.Pt (Psicastenia), 8.Sc (Schizofrenia), 9.Ma (Ipomania), 0.Si (Introversione Sociale). Si tratta delle scale più 
importanti del test in quanto indagano varie aree della personalità del soggetto (area cognitiva, area affettiva, relazioni interpersonali, meccanismi di difesa) permettendo di ricavare notevoli informazioni circa il funzionamento dell'individuo in termini sia di problematiche e difficoltà, sia di risorse personali. Esse sono le scale maggiormente corroborate da studi e ricerche. Solitamente, più una scala è elevata, maggiori saranno le difficoltà riferite dal soggetto. Elevazioni $\geq 75$ solitamente indicano la presenza di difficoltà e problematiche che si manifestano non solo da un punto di vista cognitivo ed emotivo, ma anche attraverso comportamenti disfunzionali e degni di nota. Tutti gli item su cui si basano queste scale sono compresi tra i primi 370 item del questionario;

- 15 scale di Contenuto: Anx (Ansia), Frs (Paure), Obs (Ossessione), Dep (Depressione), Hea (Preoccupazione per la salute), Biz (Pensiero bizzarro), Ang (Rabbia), Cyn (Cinismo), Asp (Comportamento antisociale), Tpa (Tipo A), Lse (Bassa autostima), Sod (Problemi sociali), Fam (Problemi familiari), Wrk (Problemi sul lavoro), Trt (Difficoltà di trattamento). Queste scale sono costituite da item il cui significato è più omogeneo, coerente ed esplicito rispetto alle scale di base. Di conseguenza, sono le scale maggiormente controllabili dal soggetto sul piano razionale essendo abbastanza chiaro ed esplicito a quali aree fa riferimento ogni item. Queste scale permettono di confermare, o meno, le informazioni emerse dalle scale di base, di chiarirle ulteriormente ed integrarle. E' possibile dividere queste scale in 4 gruppi: le scale che indagano l'eventuale presenza di sintomi specifici connessi, ad esempio, ad ansia, paure, eccessive preoccupazioni per la salute (considerando l'ordine con cui sono state presentate, sono le prime sei scale di contenuto), le scale che indagano le tendenze aggressive esterne (Ang, Cyn, Asp, Tpa), la scala che indaga l'autopercezione negativa (Lse), le scale che indagano problemi generali che il soggetto potrebbe avere con la propria famiglia, sul posto di lavoro o problematiche sociali più generiche (Sod, Fam, Wrk, Trt);

- 15 scale Supplementari: Mac-R (scala MacAndrews alcolismo rivista), Aps (Tossicodipendenza potenziale), Aas (Tossicodipendenza ammessa), O-H (Ostilità Ipercontrollata), Mds (Disagio coniugale), PK (Disturbo Post-Traumatico da stress Keane), PS (Distrubo Post-traumatico da stress Schlenger), Mt (Disadattamento universitario), A (Ansietà), R (Repressione), Es (Forza dell'Io), Do (Dominanza), Re (Responsabilità sociale), Gm (Ruolo di genere maschile), Gf (Ruolo di genere femminile). Queste scale analizzano aspetti della personalità del soggetto non indagati, o indagati solo parzialmente, dalle scale di base. Tra queste, le scale più importanti sono quelle che indagano l'eventuale presenza di utilizzo di sostanze psicotrope e di uno stile di personalità in linea con un potenziale sviluppo di una dipendenza da sostanze (Mac-R, Aps, Aas) e la scala che indaga la capacità del soggetto di gestire la frustrazione e la sua tendenza a negare difficoltà nel controllo delle emozioni e della rabbia (Ostilità Ipercontrollata).

\section{Risultati generali.}

Il primo dato significativo da sottolineare è che in tutti i grafici riassuntivi dei profili medi del campione generale non si ravvisa mai la presenza di 


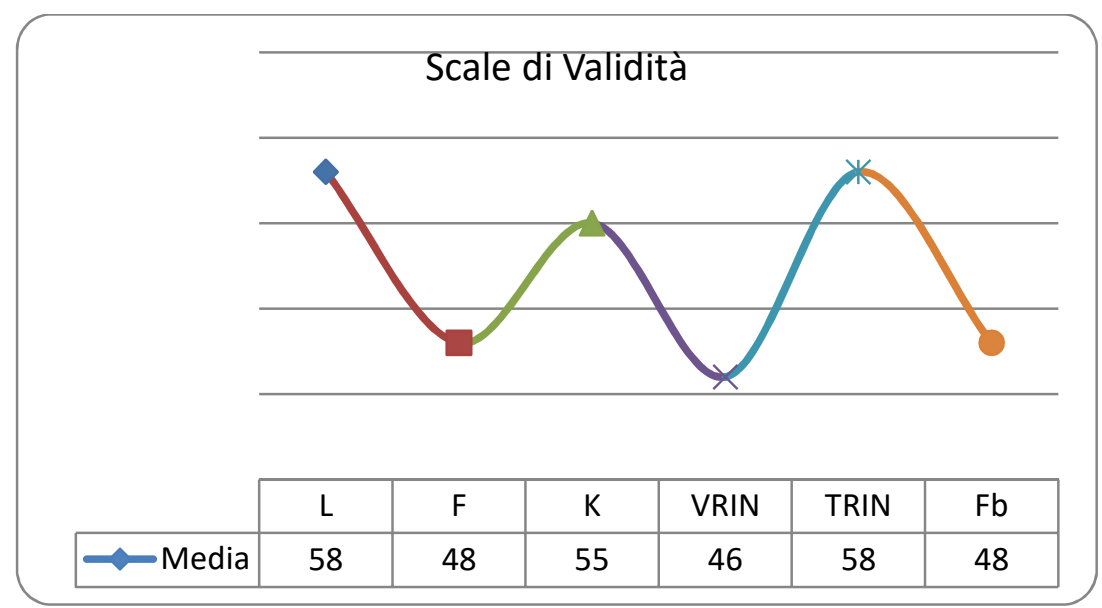

Tabella n. 1

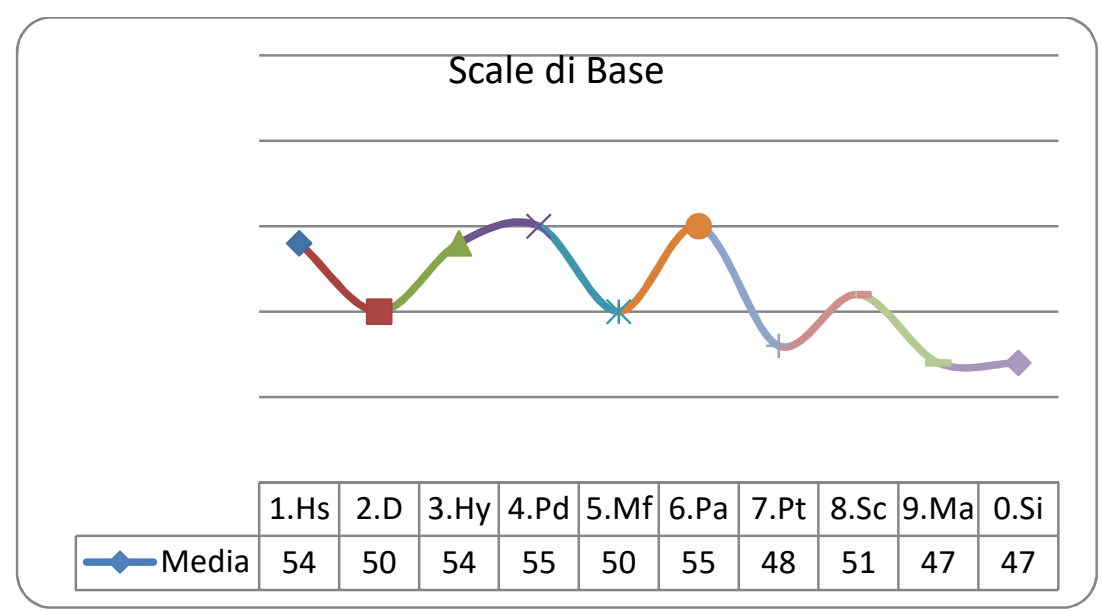

Tabella n. 2

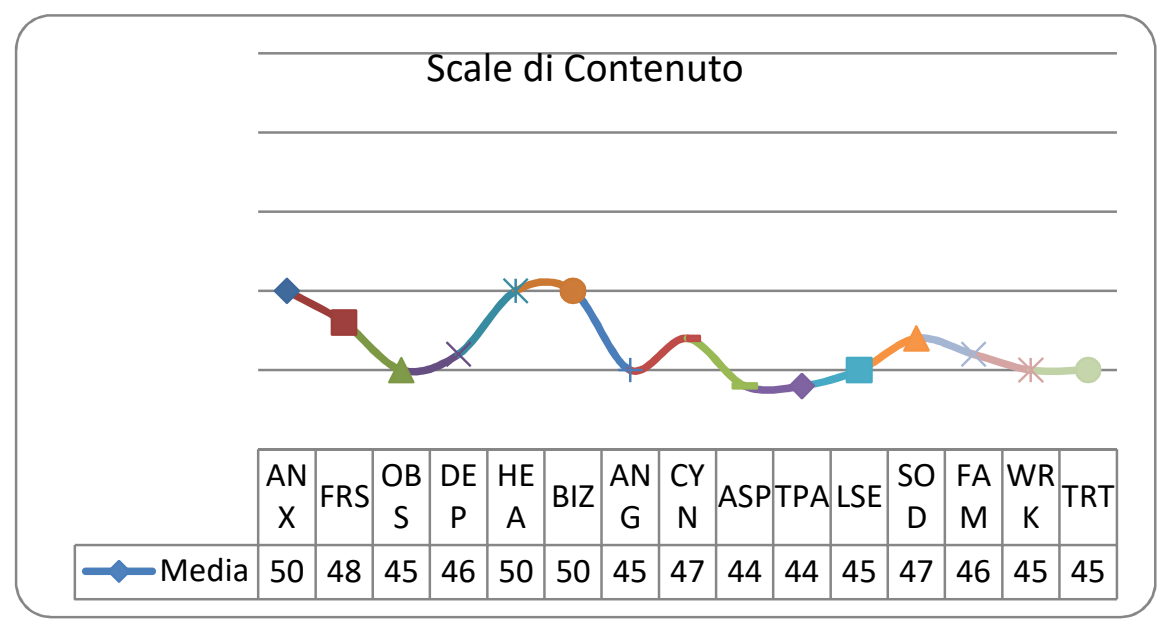

Tabella n. 3 


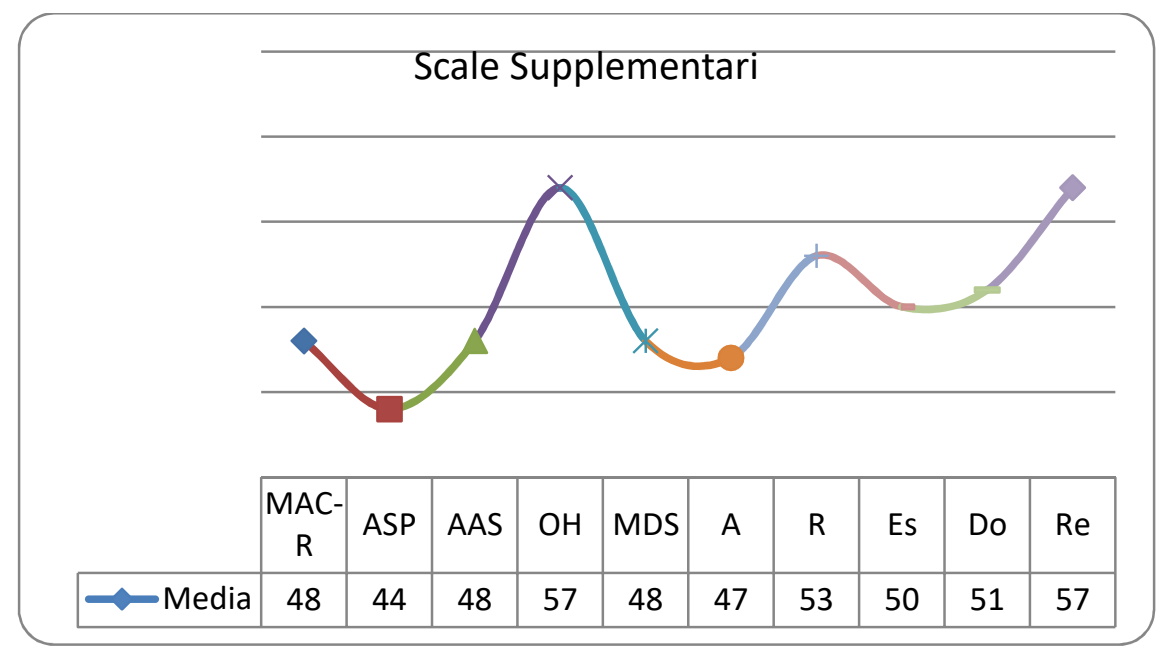

Tabella n. 4

Quanto detto conferma la sostanziale assenza di aspetti clinici patologici degni di nota nei periziandi e vale sia per il campione intero, sia per i profili medi emersi dagli uomini e dalle donne.

L'elevazione media delle scale $\mathrm{L}$ e $\mathrm{K}$ si presenta leggermente superiore a quella del campione normale, sebbene tale differenza non sia particolarmente significativa.

Di seguito verranno analizzati più in dettaglio $i$ risultati nelle varie scale (validità, base, contenuto e supplementari) con l'ausilio di tabelle che mettono a confronto i sottogruppi maschile e femminile.

\subsection{Scale di Validità.}

La tabella 5 mostra differenze rilevanti tra i sessi circa le elevazioni delle scale L e $\mathrm{K}$, con punteggi mediamente superiori nelle donne (L61, K57) rispetto agli uomini (L55, K53).

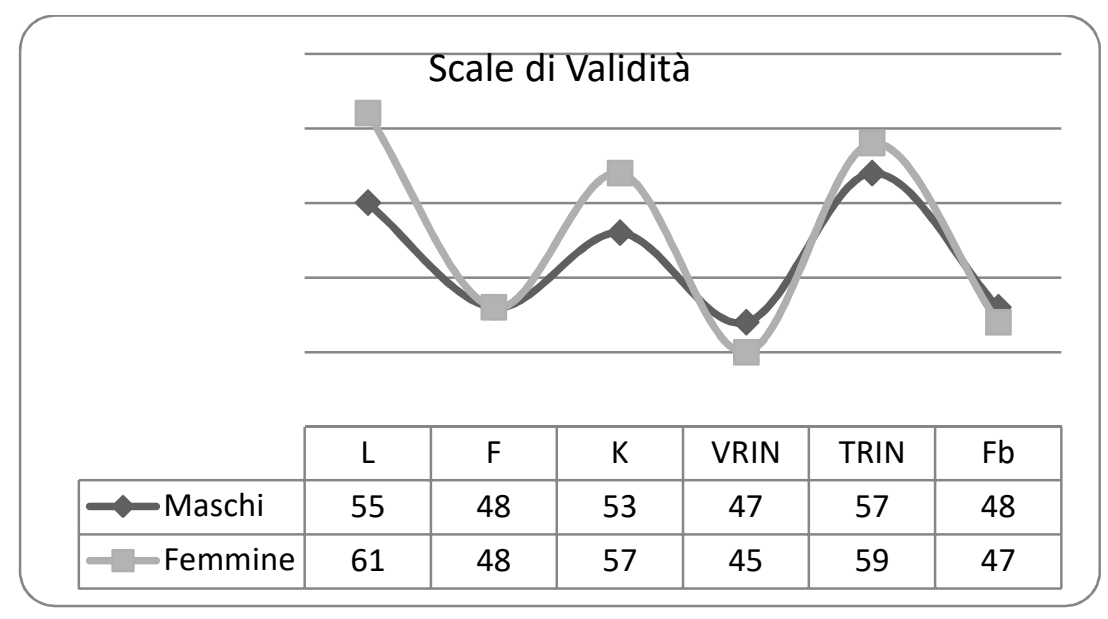

Tabella n. 5

Ciò indica che nonostante la maggior parte dei genitori presenti profili validi ed interpretabili (vadasi tabella 1), si nota nelle donne una maggiore tendenza a presentare di sé un'immagine sociale migliorativa ed anche una minore propensione a riferire le proprie difficoltà. 
$E^{\prime}$ probabile che tali atteggiamenti siano la conseguenza di un maggiore preoccupazione nelle donne di confermare la loro adeguatezza nel ruolo di madre relativamente ai canoni ed alle aspettative sociali.

I dati emersi dal nostro studio risultano parzialmente in linea con quanto presente nella letteratura: in uno studio Carr, Moretti \& Cue (16) presero in esame oltre 150 MMPI-2 somministrati a genitori in ambito di valutazione per l'affidamento dei figli ed osservarono che mediamente la scala L era più elevata nelle donne che negli uomini (63 vs 61), mentre la scala $K$ era maggiormente elevata negli uomini (55 vs 52). Altri studi precedenti invece mostravano punteggi di L e $\mathrm{K}$ mediamente più elevati nei genitori sottoposti a valutazione per l'affidamento dei figli rispetto al campione di controllo ed avevano sempre rintracciato un'elevazione mediamente maggiore della scala $\mathrm{K}$ rispetto alla scala $\mathrm{L}(\underline{17})$.

In linea con i dati emersi dallo studio di Carr et. al., nel nostro studio la scala L risulta mediamente più elevata nelle donne rispetto agli uomini ma, al contrario dello studio di Carr et al., anche la scala K risulta maggiormente elevata nelle donne. Inoltre, in linea con tutti gli studi sopra citati, nel nostro campione le elevazioni di L e K risultano superiori rispetto alle medie normative di riferimento (soprattutto nelle donne), ma nel nostro caso, in linea con Carr e differentemente dagli altri studi citati, l'elevazione media della scala $\mathrm{K}$ è inferiore all'elevazione media della scala L. Probabilmente tali differenze sono ascrivibili sia alla differente ampiezza del campione sia, soprattutto, alla differente cultura di appartenenza.

\subsection{Scale di Base.}

Nel profilo medio delle scale di Base (tabella 6) sono assenti elevazioni significative ed il profilo medio risulta sommerso (tutte le scale hanno un'elevazione $<65)$.

Osservando e confrontando tra loro i due sottogruppi (maschi e femmine) ed i punteggi medi delle scale, nei maschi la più elevata è la 6.Pa (56), seguita da 1.Hs e 3.Hy (entrambe 55), mentre nelle femmine la più elevata è la 4.Pd (56), seguita dalla 6.Pa (55).

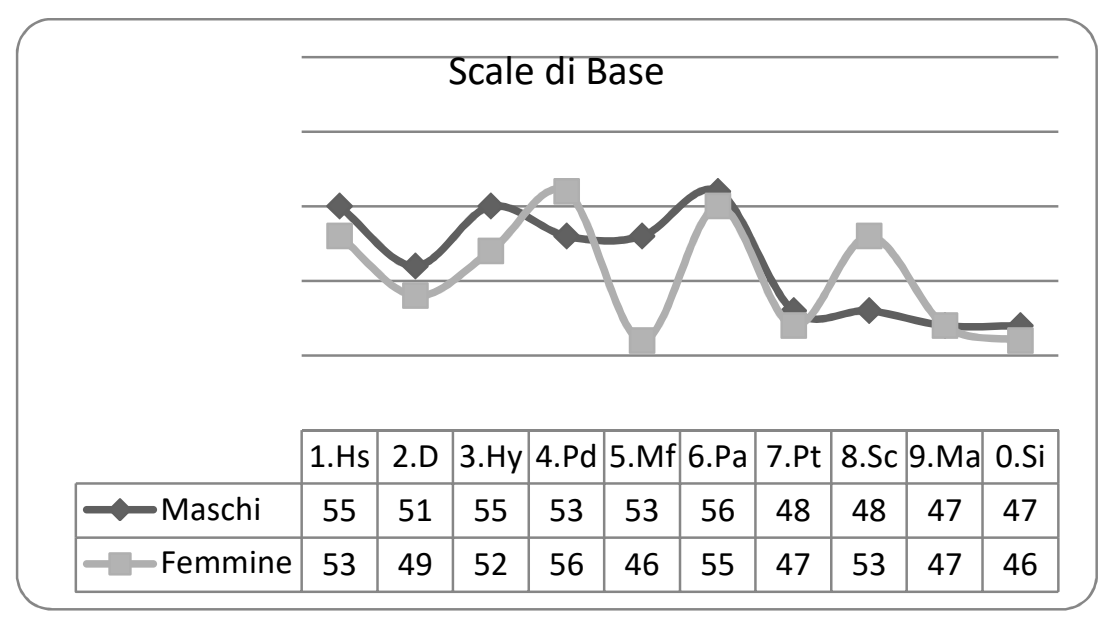

Tabella n. 6

La configurazione del profilo medio dei maschi e delle femmine, inoltre, spinge a prendere in esame 
l'eventuale presenza di alcune configurazioni tipiche, sebbene sempre sottosoglia clinicamente significativa.

Le scale che costituiscono la triade nevrotica (1.Hs, 2.D, 3.Hy), anche se mediamente più elevate negli uomini, presentano sostanzialmente lo stesso andamento in entrambi i sessi, con le scale 1 e 3 che risultano mediamente più elevate rispetto alla 2 . Tale conformazione ricorda il vallo isterico (o di Conversione), senza che però si possa affermare la sua reale esistenza a causa della scarsa elevazione delle scale 1 e 3 .

Non è inoltre presente una differenza significativa tra tali elevazioni e quella della scala 2 . Osservando singolarmente i vari protocolli, in soli 7 casi su 200 (3,5\%, di cui 4 uomini e 3 donne) si ravvisa la reale presenza di un vallo isterico.

Analoga riflessione si può fare per il campione femminile dove la conformazione delle scale 4.Pd, 5.Mf e 6.Pa è simile a quella definita vallo passivoaggressivo, anche se non è possibile affermarne la presenza a causa della scarsa elevazione delle scale 4 e 6, che non segnala componenti pulsionali o aggressive degne di nota, ed a causa del punteggio $\mathrm{T}$ della scala 5.Mf che non conferma la presenza di aspetti di passività manifesti. Osservando singolarmente i vari protocolli, solo in 3 femmine $(3 \%)$ si ravvisa la presenza di un reale vallo passivoaggressivo.

Prendendo in considerazione soltanto l'elevazione media della scala 5.Mf, si nota invece una differenza degna di nota tra $i$ sessi, con le femmine che presentano un punteggio medio più basso (M 53; F 46). Tale valore medio, tendenzialmente basso, permette di ipotizzare nelle madri passività, compiacenza, dipendenza e tendenza all'autosvalutazione. Più nel dettaglio, 28 femmine (28\%) presentano in questa scala $\mathrm{T} \leq 40$ : di conseguenza, poco più di 1 donna su 4 mostra $i$ correlati sopra citati in modo marcato.

\subsection{Analisi dei punteggi significativi $(T \geq 65)$ nel campione generale e in vari sottogruppi.}

Di seguito sono riportate varie analisi: alcune riguardano il campione totale, altre vari sottogruppi:

- considerando il campione totale ed osservando nel dettaglio i singoli test, il 35,5\% (poco più di 1/3) presenta almeno una scala di Base con punteggio significativo; di questi il $57,7 \%$ sono maschi, a conferma che le femmine mostrano un atteggiamento tendenzialmente più difeso;

- considerando separatamente il campione maschile e quello femminile, 41 maschi (41\%) e 30 femmine $(30 \%)$ presentano profili con almeno un'elevazione significativa. Butcher (18) in uno studio condotto su 1799 soggetti osservò che solo il $20 \%$ dei maschi ed il $23,5 \%$ delle femmine mostrava profili significativi (percentuali ancora più esigue di quelle osservate nel nostro studio), con andamento dei due sessi opposto a quello presente nel nostro studio in quanto erano le donne a mostrare maggiori protocolli con elevazioni significative. Ancora una volta è possibile che tali differenze siano ascrivibili alla numerosità del campione ed a differenze culturali e potranno essere verificate in studi futuri;

- considerando solo i 71 protocolli che mostrano almeno un'elevazione significativa tra le scale di Base, tra i 41 maschi il $24,4 \%$ presenta come scala più elevata la 6.Pa (mentre nel $36,6 \%$ dei casi essa risulta tra le due scale più elevate) e il $22 \%$ la $4 . P d$ (mentre nel 29,2\% dei casi essa risulta tra le due scale più elevate). Tra le 30 femmine, invece, il 26,6\% presenta come scala più elevata la 1. Hs (mentre nel 56,7\% dei casi 
essa risulta tra le due scale più elevate) e il $20 \%$ la 6.Pa (nel 36,6\% dei casi tra le due scale più elevate). Dunque, sia negli uomini sia nelle donne che mostrano nel proprio profilo elevazioni significative, la 6.Pa risulta tra le maggiormente elevate: ciò probabilmente è da ascrivere al contesto in cui tali valutazioni sono avvenute, contesto che per sua natura rende $\mathrm{i}$ periziandi maggiormente diffidenti ed inclini ad attribuire colpe e responsabilità all'esterno;

- considerando sempre i 71 protocolli con almeno un'elevazione significativa, tra i 41 maschi solo il $14,6 \%$ presenta un codice a due punte ben definito, nel 66,7\% il codice 4-6/6-4: è verosimile che tale codice in questo contesto rappresenti l'esito dei contrasti e conflitti familiari, degli attacchi e accuse tra coniugi. Tra le 30 femmine, invece, il $50 \%$ presenta un codice a due punte definito, solitamente il codice 1-3/3-1 o 1-2/2-1. Le scale di questi ultimi due codici fanno parte tutte della triade nevrotica e hanno fattori in comune come, ad esempio: ansia, preoccupazioni per la salute, richieste di attenzioni e supporto; il primo è più tipico di chi tende alla conversione somatica, alla repressione delle emozioni di rabbia e dell'aggressività, alla dipendenza affettiva, a comportamenti di estroversione superficiale, egocentrici e manipolativi; il secondo, invece, è tipico di chi presenta notevoli problemi somatici o, comunque, tende ad essere eccessiva attenzione ai segnali provenienti dal corpo con reazioni esagerate al minimo segnale fisico, apparendo ansioso, rimuginante, teso, facilmente irritabile ed infelice.

\subsection{Scale di Contenuto.}

Dal grafico delle scale di Contenuto (tabella 7) non si evidenziano differenze degne di nota tra $i$ due sessi.

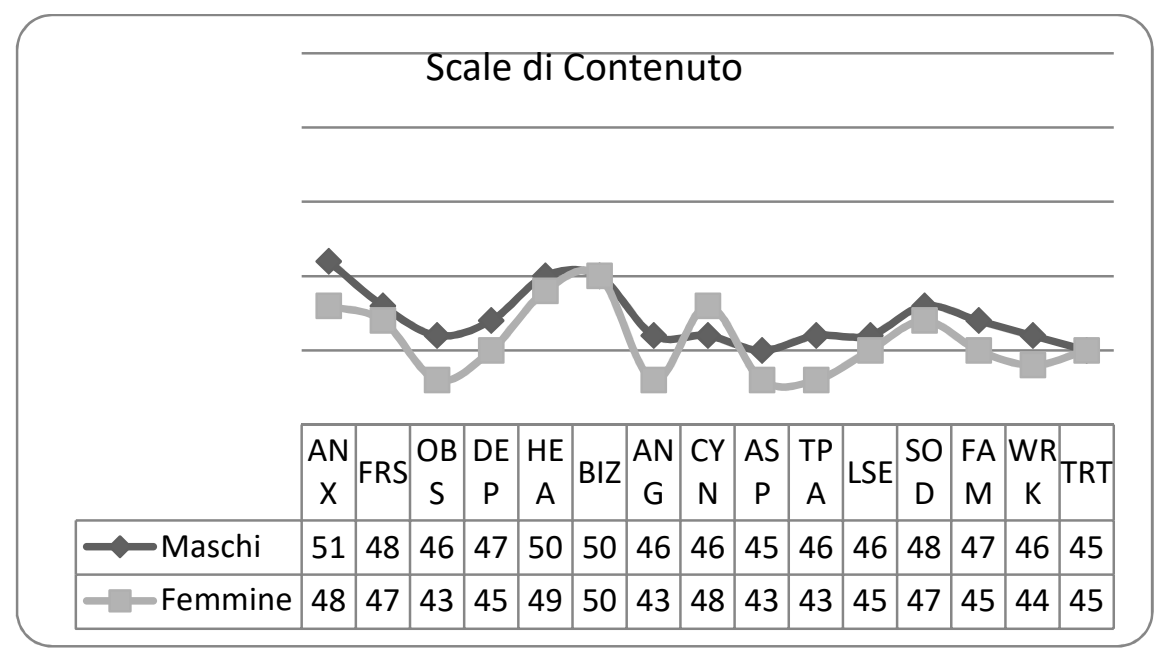

Tabella n. 7

\subsection{Scale Supplementari.}

Tra le scale Supplementari (tabella 8) si nota una differenza circa l'elevazione media della scala $\mathrm{O}-\mathrm{H}$ (Ostilità Ipercontrollata) (M54, F60) tra i due sessi. 


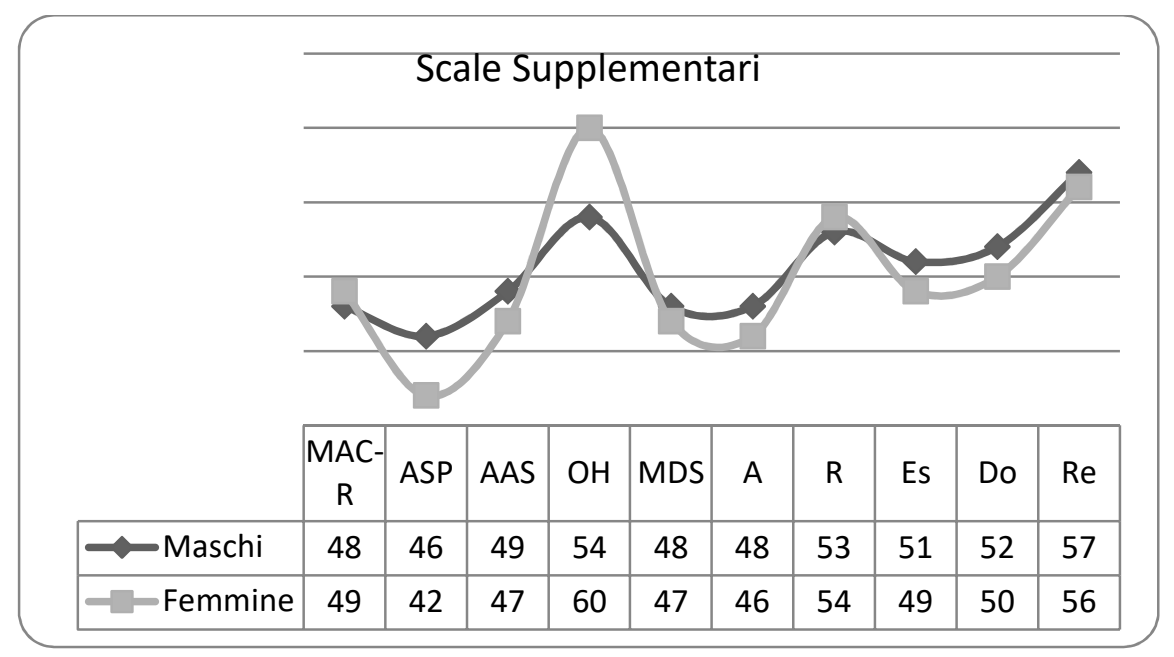

Tabella n. 8

Mentre nei maschi non si notano particolarità, nel profilo medio delle femmine la scala $\mathrm{O}-\mathrm{H}$ mostra un'elevazione che, seppur non significativa, è degna di nota. Inoltre, analizzando i singoli protocolli, 25 femmine $(25 \%)$ presentano in questa scala un elevazione $\mathrm{T} \geq 65$, mentre sono ben 59 (59\%) le femmine che hanno un punteggio $\mathrm{T} \geq 60$.

Tale scala fu sviluppata con $\mathrm{i}$ detenuti in ambito carcerario da Megargee, Cook e Mendelsohn (1967) allo scopo di identificare chi, pur mostrando difficoltà nell'esprimere rabbia apertamente e comportandosi generalmente in modo ipercontrollato, poteva avere (anche se raramente)

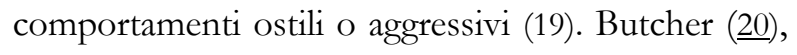
però, sottolinea che al di fuori delle popolazioni carcerarie le elevazioni significative di tale scala sono l'esito della tendenza a negare intenzioni o azioni ostili. Alla luce di quest'ultimo significato, l'elevazione media maggiore nelle femmine risulta in linea con quanto già emerso dalle scale di validità, con tendenza a migliorare la propria immagine di sé, a non riferire le proprie difficoltà ed a negare, soffocare e reprimere intenzioni ed azioni ostili.

\section{Conclusioni.}

In generale nel nostro studio le scale L e K si presentano mediamente più elevate rispetto al campione normativo, con una differenza non degna di nota rispetto alla popolazione generale. Ciò disattende l'ipotesi attesa di una più attiva difensività già rilevata da altri studi in letteratura (21). Un possibile fattore che può aver inciso, almeno in parte, è che i questionari sono stati tutti somministrati dopo un colloquio iniziale e il proiettivo di Rorschach, con probabile riduzione dei vissuti di ansia e relativo abbassamento dello schermo difensivo.

Anche per quanto riguarda tutte le altre scale del profilo medio (Base, Contenuto e Supplementari) non si ravvisano nel campione totale elevazioni medie significative.

Dal confronto tra $i$ sottogruppi maschile e femminile, tuttavia, sono emerse alcune differenze significative.

Le madri hanno ottenuto punteggi medi più elevati nelle scale $\mathrm{L}$ e $\mathrm{K}$ rispetto ai padri, con $\mathrm{L}$ moderatamente elevata (L 61) e più alta della $K$. Tale dato suggerisce che sia presente nelle donne una tendenza, più sensibile rispetto ai maschi, volontaria ed abbastanza esplicita, a cercare di modificare in senso migliorativo la propria immagine sociale, come potrebbero indicare anche $\mathrm{i}$ punteggi tendenzialmente più bassi della scala di 
Base 5.Mf e più elevati della scala Supplementare OH. Tuttavia, anche nelle donne, i profili che emergono dal questionario risultano generalmente validi, con rappresentazioni abbastanza attendibili delle loro reali caratteristiche di personalità.

Pertanto, nonostante questo studio presenti alcuni limiti, quali la numerosità circoscritta del campione che non può quindi considerarsi rappresentativo delle coppie in fase di separazione e l'assenza di un gruppo di controllo specifico, emergono comunque alcune importanti indicazioni di carattere generale che possono aiutare lo psicodiagnosta ad orientarsi nel suo lavoro di valutazione con i periziandi. In tal senso i profili indicano globalmente l'assenza sia di stili difensivi di risposta così significativi da inficiare la validità delle informazioni, sia una sostanziale normalità, ossia l'assenza di sintomatologia clinica attiva al momento della prova, contrariamente a quanto si potrebbe ipotizzare sulla base della fase vitale particolarmente stressante che accompagna questi soggetti.

Dall'analisi più dettagliata dei nostri profili, meno della metà del campione (41 maschi e 30 femmine pari al 35,5\%) ha presentato almeno una scala di Base con un'elevazione significativa: il minor numero di femmine risulta essere coerente con la presenza di un atteggiamento tendenzialmente più difeso e sensibile al giudizio altrui. Tale dato non è in linea con uno studio di Butcher (22) in cui egli osservò percentuali ancora più esigue di profili non sommersi ed una maggioranza di essi negli uomini, differenza questa probabilmente ascrivibile alla differente numerosità ed alla cultura di appartenenza dei campioni.

Considerando solo i 71 protocolli con almeno un'elevazione significativa tra le scale di Base, nel $24,4 \%$ degli uomini la scala maggiormente elevata è stata la 6.Pa, mentre nel $22 \%$ la 4.Pd. Nelle donne nel 26,6\% dei casi la scala più elevata è stata la 1. Hs, mentre nel 20\% la 6.Pa. Dunque, nei profili che presentano elevazioni significative, a prescindere dal sesso, la $\mathrm{Pa}$ è risultata tra le scale più elevate.

Analizzando sempre i 71 protocolli con almeno un'elevazione significativa, tra i 41 maschi solo nel $14,6 \%$ è stato possibile rintracciare la presenza di un codice a due punte definito, e nel 66,7\% si è trattato del 4-6/6-4; mentre tra le 30 femmine, nel 50\% i codici a due punte definiti sono stati il 1-3/3-1 e 12/2-1.

L'obiettivo del nostro studio era essenzialmente esplorativo. Riteniamo comunque importanti le indicazioni qui emerse in via preliminare e future ricerche potranno essere impostate per approfondirle in modo più attendibile.

\section{Note.}

(1). Cicioni R., Valgimigli S., Donati C., Caravelli T., "Analisi qualitativa di 20 protocolli Rorschach di soggetti con tentato suicidio", Rivista sperimentale di Freniatria, 2016.

(2). Ibidem.

(3). Cicioni R., Caravelli T., Patruno M., Lasala M., "L'utilizzo dei test all'interno delle consulenze tecniche di ufficio per l'affido con particolare riferimento al Rorschach", Notiziario dell'Ordine degli Psicologi della Regione Puglia, n. 17, 2016, pp. 109-113.

(4). Fielding N. G., Fielding J. L., Linking data: The articulation of qualitative and quantitative methods in social research, Sage, 1986.

(5). Montesarchio G., Colloquio da Manuale, Giuffrè, Milano, 1998.

(6). LaFortune K.A. \& Carpenter B.N., "Custody evaluations: A Survey of mental health Professionals", Behavioral Sciences and the Law, 1998; Quinnell F.A., Bow J.N., "Psychological tests used in child custody evaluations", Behavioral Sciences and the Law, 2001.

(7). Quinnell F.A., Bow J.N., op. cit.; Otto R.K., "Use of the MMPI-2 in Forensic Settings", Mental Healt Law \& Policy Faculty Publications, 2002.

(8). Cicioni R., Caravelli T., "I due strumenti della valutazione diagnostica attraverso il Rorschach: l'esaminatore e il test", Psicoin - Rivista dell'Ordine degli Psicologi della Marche, a. XVIII, n. 2, Settembre 2012.

(9). Graham J. R., Watts D., Timbrook R.E., "Detecting fakegood and fake-bad MMPI-2 profiles", Journal of Personality Assessment, n. 57, 1991, pp. 264-277.

(10). Sirigatti S., Giannini M., "Identificazione del comportamento distorsivo (faking good) con il MMPI-2: caratteristiche psicometriche della scala S”, Bollettino di Psicologia Applicata, 47(232), 2000, pp. 61-69. 
(11). Marzioni B., Sardella L., "Utilizzo del MMPI-2 nei casi di affido: profilo dissimulatorio e profilo difensivo", Items del testing psicologico, 2006.

(12). Graham J. R., MMPI-2: Assessing Personality and Psychopathology (3rd ed.), Oxford University Press, 1999.

(13). Sirigatti S., Stefanile C., MMPI-2: Aggiornamento all'adattamento italiano Scale di validità, Harris-Lingoes, supplementari, di contenuto e PSY-5, Giunti O.S., Firenze, 2011.

(14). Pancheri P., Sirigatti S., "L'adattamento italiano del MMPI-2", in Hathaway S.R., McKinley J.C. (eds.), MMPI-2 - Manuale, OS, Firenze, 1995.

(15). Caporale R., MMPI-2, MMPI-2 RE e MMPI-2 RF. Guida pratica all'uso dei test in ambito clinico e giuridicoperitale, FrancoAngeli, Milano, 2013 (17);

(16) Carr G.D., Moretti M.M., Cue B.J.H., "Evaluating Parenting Capacity: Validity Problems With the MMPI-2, PAI, CAPI, and Ratings of Child Adjustment", Professional Psychology: Research and Practice, 2005.

(17). Siegel J.C., "Traditional MMPI-2 validity indicators and initial presentation in custody evaluations", American Journal of Forensic Psychology, 1996; Bathurst K., Gottfried A.W. \& Gottfried A. E., "Normative data for the MMPI-2 in child custody litigation", Psychological Assessment, 1997; Posthuma A. B. \& Harper J. F., "Comparison of MMPI-2 responses of child custody and personal injury litigants", Professional Psychology: Research and Practice, 1998; Bagby R. M., Nicholson R. A., Buis T., Radovanovic H. \& Fidler B. J., "Defensive responding on the MMPI-2 in family custody and access evaluations", Psychological Assessment, 1999.

(18). Butcher J. N., Frequency of MMPI-2 scores in forensic evaluations. Paper presented at the $32^{\text {nd }}$ annual Symposium on Recent Developments in the use of the MMPI-2, Minneapolis, MN, 1997.

(19). Butcher J. N., Williams C.L., Fondamenti per l'interpretazione del MMPI-2 e del MMPI-A, Giunti O.S., Firenze, 1996.

(20). Ibidem.

(21). Siegel J.C., "Traditional MMPI-2 validity indicators and initial presentation in custody evaluations", American Journal of Forensic Psychology, 1996; Bathurst K., Gottfried A.W. \& Gottfried A. E., "Normative data for the MMPI-2 in child custody litigation", Psychological Assessment, 1997; Posthuma A. B. \& Harper J. F., "Comparison of MMPI-2 responses of child custody and personal injury litigants", Professional Psychology: Research and Practice, 1998; Bagby R. M., Nicholson R. A., Buis T., Radovanovic H. \& Fidler B. J., "Defensive responding on the MMPI-2 in family custody and access evaluations", Psychological Assessment, 1999; Carr G.D., Moretti M.M., Cue B.J.H., "Evaluating Parenting Capacity: Validity Problems With the MMPI-2, PAI, CAPI, and Ratings of Child Adjustment”, Professional Psychology: Research and Practice, 2005.

(22). Butcher J. N., Frequency of MMPI-2 scores in forensic evaluations. Paper presented at the $32^{\text {nd }}$ annual Symposium on Recent Developments in the use of the MMPI-2, Minneapolis, MN, 1997.

\section{Bibliografia.}

- Bagby R. M., Nicholson R. A., Buis T., Radovanovic H. \& Fidler B. J., "Defensive responding on the MMPI-2 in family custody and access evaluations", Psychological Assessment, 1999.

- Bathurst K., Gottfried A.W. \& Gottfried A. E., "Normative data for the MMPI-2 in child custody litigation", Psychological Assessment, 1997.

- Berto D. (a cura di), MMPI, MMPI-2 e MMPI- $A$ in tribunale. Manuale pratico per consulenti tecnici, avvocati e giudici con casistica criminologica italiana, Ed. Giunti O.S., Firenze, 2006.

- Butcher J. N, MMPI-2 in psychological treatment, Oxford University Press, 1990.

- Butcher J. N., Frequency of MMPI-2 scores in forensic evaluations. Paper presented at the $32^{\text {nd }}$ annual Symposium on Recent Developments in the use of the MMPI-2, Minneapolis, MN, 1997.

- Butcher J. N., Graham J. R., Tellegen A., Kaemmer B., Manual for the restandardized Minnesota Multiphasic Personality Inventory: MMPI2. An administrative and interpretative guide, University of Minnesota Press, 1989.

- Butcher J. N., Williams C.L., Fondamenti per l'interpretarione del MMPI-2 e del MMPI-A, Giunti O.S., Firenze, 1996.

- Caporale R., MMPI-2, MMPI-2 RE e MMPI-2 RF. Guida pratica all'uso dei test in ambito clinico e giuridico-peritale, FrancoAngeli, Milano, 2013.

- Carr G.D., Moretti M.M., Cue B.J.H., "Evaluating Parenting Capacity: Validity Problems With the MMPI-2, PAI, CAPI, and Ratings of Child Adjustment", Professional Psychology: Research and Practice, 2005.

- Caviglia G., Del Castello E., La diagnosi in psicologia clinica, FrancoAngeli, Milano, 2008.

- Cicioni R., Caravelli T., "I due strumenti della valutazione diagnostica attraverso il Rorschach: l'esaminatore e il test", Psicoin - Rivista dell'Ordine degli Psicologi della Marche, a. XVIII, n. 2, Settembre 2012.

- Cicioni R., Caravelli T., Patruno M., Lasala M., "L'utilizzo dei test all'interno delle consulenze tecniche di ufficio per l'affido con particolare riferimento al Rorschach", Notiziario dell'Ordine degli Psicologi della Regione Puglia, n. 17, 2016, pp. 109-113.

- Cicioni R., Valgimigli S., Donati C., Caravelli T., "Analisi qualitativa di 20 protocolli Rorschach di soggetti con tentato suicidio", Rivista sperimentale di Freniatria, 2016.

- Fielding N. G., Fielding J. L., Linking data: The articulation of qualitative and quantitative methods in social research, Sage, 1986.

- Graham J. R., Watts D., Timbrook R.E., "Detecting fake-good and fake-bad MMPI-2 
profiles", Journal of Personality Assessment, n. 57, 1991, pp. 264-277.

- Graham J. R., MMPI-2: Assessing Personality and Psychopathology (3rd ed.), Oxford University Press, 1999.

- Hathaway S. R., McKinley J. C., Manual for the Minnesota Multiphasic Personality Inventory, University of Minnesota Press, 1942.

- LaFortune K.A. \& Carpenter B.N., "Custody evaluations: A Survey of mental health Professionals", Behavioral Sciences and the Law, 1998.

- Marzioni B., Sardella L., "Utilizzo del MMPI-2 nei casi di affido: profilo dissimulatorio e profilo difensivo", Items del testing psicologico, 2006.

- Megargee E. I., Cook P. E., Mendelshon G. A., "Development and a validation of an MMPI scale of assaultiveness in overcontrolled individuals", Journal of Abnormal Psychology, n. 72, 1967, pp. 519-528.

- Montesarchio G., Colloquio da Manuale, Giuffrè, Milano, 1998.
- Otto R.K., "Use of the MMPI-2 in Forensic Settings", Mental Healt Law \& Policy Faculty Publications, 2002.

- Pancheri P., Sirigatti S., "L'adattamento italiano del MMPI-2", in Hathaway S.R., McKinley J.C. (eds.), MMPI-2 - Manuale, OS, Firenze, 1995.

- Posthuma A. B. \& Harper J. F., "Comparison of MMPI-2 responses of child custody and personal injury litigants", Professional Psychology: Research and Practice, 1998.

- Quinnell F.A., Bow J.N., "Psychological tests used in child custody evaluations", Behavioral Sciences and the Law, 2001.

- Saraceni C., Montesarchio G., Introdurione alla psicodiagnostica, NIS, Roma, 1988.

- Siegel J.C., "Traditional MMPI-2 validity indicators and initial presentation in custody evaluations", American Journal of Forensic Psychology, 1996.

- Sirigatti S., Giannini M., "Identificazione del comportamento distorsivo (faking good) con il MMPI-2: caratteristiche psicometriche della scala S", Bollettino di Psicologia Applicata, 47(232), 2000, pp. 61-69.

- Sirigatti S., Stefanile C., MMPI-2: Aggiornamento all'adattamento italiano Scale di validità, HarrisLingoes, supplementari, di contenuto e PSY-5, Giunti O.S., Firenze, 2011. 